PROCEEDINGS OF THE

AMERICAN MATHEMATICAL SOCIETY

Volume 126, Number 2, February 1998, Pages 491-497

S 0002-9939(98)04048-9

\title{
ON THE EQUIVALENCE OF A THEOREM OF KISYŃSKI AND THE HILLE-YOSIDA GENERATION THEOREM
}

\author{
WOJCIECH CHOJNACKI
}

(Communicated by Palle E. T. Jorgensen)

\begin{abstract}
We show that a theorem of Kisyński on the generation of Banachalgebra homomorphisms of certain convolution algebras is equivalent to the Hille-Yosida theorem on the generation of operator-valued one-parameter semigroups.
\end{abstract}

\section{INTRODUCTION}

Let $\mathbb{F}$ be either the field $\mathbb{R}$ of real numbers or the field $\mathbb{C}$ of complex numbers, and let $\mathbb{R}^{+}$be the set of all positive numbers. For each $\lambda \in \mathbb{R}$, denote by $\epsilon_{\lambda}$ the function

$$
\epsilon_{\lambda}(t)=e^{\lambda t} \quad\left(t \in \mathbb{R}^{+}\right) .
$$

Let $\omega$ be a non-negative number. Let $L_{\omega}^{1}\left(\mathbb{R}^{+}, \mathbb{F}\right)$ be the space of equivalence classes (under equality almost everywhere) of Lebesgue measurable $\mathbb{F}$-valued functions $f$ on $\mathbb{R}^{+}$for which $|f| \epsilon_{\omega}$ is Lebesgue integrable. With the addition and scalar multiplication derived from the pointwise addition and scalar multiplication of the functions, and with the norm given by

$$
\|f\|_{1, \omega}=\int_{\mathbb{R}^{+}}|f(t)| e^{\omega t} d t
$$

(where the same symbol $f$ is used to denote both a function and its equivalence class $), L_{\omega}^{1}\left(\mathbb{R}^{+}, \mathbb{F}\right)$ is a Banach space over $\mathbb{F}$. With convolution

$$
(f * g)(t)=\int_{0}^{t} f(t-s) g(s) d s \quad\left(\text { a.e. } t \in \mathbb{R}^{+}\right)
$$

as the product, it becomes a Banach algebra over $\mathbb{F}$. When $\mathbb{F}$ is understood, $L_{\omega}^{1}\left(\mathbb{R}^{+}, \mathbb{F}\right)$ will be abbreviated to $L_{\omega}^{1}\left(\mathbb{R}^{+}\right)$.

Hereafter all Banach algebras will be assumed to be over $\mathbb{F}$. Any particular choice of the ground field $\mathbb{F}$ will be inessential for the validity of results.

Let $\boldsymbol{A}$ be a Banach algebra (with identity or not). An indexed family $\left\{r_{\lambda}\right\}_{\lambda \in U}$ of elements of $\boldsymbol{A}$, where $U$ is a subset of $\mathbb{F}$, is called a pseudo-resolvent if the following

Received by the editors April 22, 1996 and, in revised form, August 12, 1996.

1991 Mathematics Subject Classification. Primary 46J25; Secondary 43A20, 47A10, 47D03.

Key words and phrases. Banach algebra, convolution algebra, one-parameter semigroup, representation, resolvent.

(C)1998 American Mathematical Society 
Hilbert equation is satisfied:

$$
r_{\lambda}-r_{\mu}=(\mu-\lambda) r_{\lambda} r_{\mu} \quad(\lambda, \mu \in U) .
$$

Direct verification shows that, for each non-negative number $\omega,\left\{\epsilon_{-\lambda}\right\}_{\lambda \in(\omega,+\infty)}$ is a pseudo-resolvent in $L_{\omega}^{1}\left(\mathbb{R}^{+}\right)$satisfying

$$
\left\|\epsilon_{-\lambda}^{* n}\right\|_{1, \omega}=\left\|\epsilon_{-\lambda}\right\|_{1, \omega}^{n}=\frac{1}{(\lambda-\omega)^{n}} \quad(\lambda \in(\omega,+\infty), n \in \mathbb{N}),
$$

where

$$
f^{* n}=\underbrace{f * \cdots * f}_{n \text { times }}
$$

for any $f \in L_{\omega}^{1}\left(\mathbb{R}^{+}\right)$. For a class of pseudo-resolvents defined over $(\omega,+\infty)$ and satisfying a growth condition resembling (2) (namely condition (i) from Theorem 1 stated below), $\left\{\epsilon_{-\lambda}\right\}_{\lambda \in(\omega,+\infty)}$ is universal. This is a consequence of the following result of J. Kisyński [4]:

Theorem 1 (Kisyński). Let $\boldsymbol{A}$ be a Banach algebra, let $\omega$ be a non-negative number, let $\left\{r_{\lambda}\right\}_{\lambda \in(\omega,+\infty)}$ be a pseudo-resolvent in $\boldsymbol{A}$, and let

$$
M=\sup \left\{(\lambda-\omega)^{n}\left\|r_{\lambda}^{n}\right\|: n \in \mathbb{N}, \lambda \in(\omega,+\infty)\right\} .
$$

Then the following conditions are equivalent:

(i) $M<+\infty$;

(ii) there exist a continuous homomorphism $T: L_{\omega}^{1}\left(\mathbb{R}^{+}\right) \rightarrow \boldsymbol{A}$ such that $T\left(\epsilon_{-\lambda}\right)=$ $r_{\lambda}$ for each $\lambda \in(\omega,+\infty)$.

Furthermore, if a continuous homomorphism $T: L_{\omega}^{1}\left(\mathbb{R}^{+}\right) \rightarrow \boldsymbol{A}$ satisfying $T\left(\epsilon_{-\lambda}\right)=$ $r_{\lambda}$ for each $\lambda \in(\omega,+\infty)$ exists, then it is unique and $\|T\|=M$.

The original statement of the above theorem deals only with the case $\omega=0$, but generalisation to the case $\omega>0$ is straightforward. The theorem has a number of interesting applications that include: $1^{\circ}$ a result concerning the generation of one-parameter integrated semigroups; $2^{\circ}$ a result concerning the generation of a one-parameter semigroup, acting on the bidual $E^{* *}$ of a Banach space $E$, such that the semigroup trajectories passing through elements of the $*$-weak sequential closure of $E$ in $E^{* *}$ are $*$-weakly Borel measurable; $3^{\circ}$ a generalisation of the Trotter-Kato theorem on the convergence of sequences of one-parameter semigroups. Kisyński has given two proofs of Theorem 1. One of them is straightforward and relies on modification of the approximation argument used by K. Yosida in his proof of the Hille-Yosida theorem. The other of Kisyński's proofs is more involved. It is based on an idea originally conceived by W. Arendt and further pursued by B. Henning and F. Neubrander (cf. $[1,3,5]$ ), and draws upon a characterisation, due to D. V. Widder, of the Laplace transforms of functions in $L^{\infty}\left(\mathbb{R}^{+}\right)$.

The aim of this note is to show that Theorem 1 can be derived directly from the Hille-Yosida theorem. As shown by Kisyński, the Hille-Yosida theorem is a consequence of Theorem 1. Both theorems are therefore equivalent. Note that the Hille-Yosida theorem, concerning the generation of homomorphisms defined on the additive semigroup $[0,+\infty)$ and taking values in the algebra of all linear continuous operators on a Banach space, does not directly extend to deal with homomorphisms taking values in an arbitrary Banach algebra. It is only by changing the domain of homomorphisms from $[0,+\infty)$ to $L_{\omega}^{1}\left(\mathbb{R}^{+}\right)$, as done in Theorem 1 , that the case of Banach-algebra homomorphisms can be successfully treated. 


\section{A RESUlt on the REgUlar REPRESENTAtion}

We begin by presenting a simple but useful result from the theory of representations of Banach algebras.

Let $\boldsymbol{A}$ be a commutative Banach algebra. A net $\left\{e_{\lambda}\right\}_{\lambda \in \Lambda}$ in $\boldsymbol{A}$, where $\Lambda$ is a directed set, is an approximate identity for $\boldsymbol{A}$ if

$$
\lim _{\lambda \in \Lambda} e_{\lambda} x=x \quad(x \in \boldsymbol{A}) .
$$

An approximate identity $\left\{e_{\lambda}\right\}_{\lambda \in \Lambda}$ will be termed metric if

$$
\limsup _{\lambda \in \Lambda}\left\|e_{\lambda}\right\| \leq 1 \text {. }
$$

Let $\mathcal{L}(\boldsymbol{A})$ be the Banach algebra of all bounded linear operators from $\boldsymbol{A}$ into itself with the norm

$$
\|T\|=\sup _{\|x\| \leq 1}\|T x\| \quad(T \in \mathcal{L}(\boldsymbol{A})) .
$$

For each $x \in A$, let $\rho_{x}$ be the operator in $\mathcal{L}(\boldsymbol{A})$ given by

$$
\rho_{x} y=x y \quad(y \in \boldsymbol{A}) .
$$

It is readily verified that the mapping $\rho: x \mapsto \rho_{x}$ is a homomorphism of $\boldsymbol{A}$ into $\mathcal{L}(\boldsymbol{A})$. It is called the regular representation of $\boldsymbol{A}$ on $\boldsymbol{A}$. Denote by $\rho_{\boldsymbol{A}}$ the image of $\boldsymbol{A}$ via $\rho$. We can now prove the following result:

Proposition 1. Let $\boldsymbol{A}$ be a commutative Banach algebra possessing a metric approximate identity. Then $\rho$ is an isometry and $\rho_{\boldsymbol{A}}$ is a closed subalgebra of $\mathcal{L}(\boldsymbol{A})$.

Proof. For each $x \in \boldsymbol{A}$, we have

$$
\left\|\rho_{x}\right\|=\sup _{\|y\| \leq 1}\|x y\| \leq\|x\| .
$$

Let $\left\{e_{\lambda}\right\}_{\lambda \in \Lambda}$ be a metric approximate identity for $\boldsymbol{A}$. Clearly, for each $\lambda \in \Lambda$,

$$
\left\|\rho_{x} e_{\lambda}\right\| \leq\left\|\rho_{x}\right\|\left\|e_{\lambda}\right\| \text {. }
$$

Rewriting (4) as $\lim _{\lambda \in \Lambda} \rho_{x} e_{\lambda}=x$, we see that

$$
\|x\| \leq\left\|\rho_{x}\right\| \liminf _{\lambda \in \Lambda}\left\|e_{\lambda}\right\|
$$

and further, in view of (5),

$$
\|x\| \leq\left\|\rho_{x}\right\| .
$$

Combining the latter inequality with (6), we find that $\rho$ is an isometry. Since $\boldsymbol{A}$ is complete, so too is its isometric image $\rho_{\boldsymbol{A}}$. Now, since $\mathcal{L}(\boldsymbol{A})$ is complete, $\rho_{\boldsymbol{A}}$ is closed in $\mathcal{L}(\boldsymbol{A})$.

Note that condition (5) appearing in the definition of a metric approximate identity can replaced by the condition

$$
\lim _{\lambda \in \Lambda}\left\|e_{\lambda}\right\|=1,
$$

which perhaps better justifies the qualification 'metric'. Indeed, if we let $x$ be any non-zero element of $\boldsymbol{A}$, then (6) and (7) imply that

$$
\liminf _{\lambda \in \Lambda}\left\|e_{\lambda}\right\| \geq 1
$$

for every approximate identity $\left\{e_{\lambda}\right\}_{\lambda \in \Lambda}$. Now the equivalence of (5) and (8) is obvious. 


\section{RENORMing BANACH ALGEBRAS}

In this section, we establish a theorem about renorming Banach algebras, which will be useful in the subsequent considerations.

Theorem 2. Let $\boldsymbol{A}$ be a commutative Banach algebra, let $\omega$ be a non-negative number, and let $\left\{r_{\lambda}\right\}_{\lambda \in(\omega,+\infty)}$ be a pseudo-resolvent in $\boldsymbol{A}$ for which $M$ defined in (3) is finite. Then there exists a submultiplicative norm $|\|\cdot \mid\|$ on $\boldsymbol{A}$ such that

$$
\|x\| \leq\|x\| \leq M\|\| x\|\| \quad(x \in \boldsymbol{A})
$$

and

$$
\left\|\left|r_{\lambda} \|\right| \leq \frac{1}{\lambda-\omega} \quad(\lambda \in(\omega,+\infty))\right.
$$

Proof. Fix $\lambda \in(\omega,+\infty)$ arbitrarily. Set

$$
s_{\lambda}=(\lambda-\omega) r_{\lambda}
$$

Borrowing an idea from [2], for each $x \in \boldsymbol{A}$, consider a representation of $x$ in the form

$$
x=\sum_{k=0}^{\infty} a_{k} s_{\lambda}^{k}
$$

where $a_{k} \in \boldsymbol{A}$ and only finitely many $a_{k}$ are different from zero. Note that while $\boldsymbol{A}$ might not have an identity and while $s_{\lambda}^{0}$ standing alone might have no obvious meaning, we can always interpret $a_{0} s_{\lambda}^{0}$ as $a_{0}$. Set

$$
\|x\|_{\lambda}=\inf \sum_{k=0}^{\infty}\left\|a_{k}\right\|
$$

where the infimum is taken over all possible representations of $x$ in the form (11). It is plain that $\left\|\left|s_{\lambda}\right|\right\|_{\lambda} \leq 1$, or equivalently

$$
\left\|\left|r_{\lambda}\right|\right\|_{\lambda} \leq \frac{1}{\lambda-\omega}
$$

We claim that $\||\cdot|\|_{\lambda}$ is a submultiplicative pseudonorm. Indeed, if $y=\sum_{k=0}^{\infty} b_{k} s_{\lambda}^{k}$, then $x y=\sum_{k=0}^{\infty} c_{k} s_{\lambda}^{k}$, where $c_{k}=\sum_{l=0}^{k} a_{k-l} b_{l}$. Hence

$$
\|x y\|_{\lambda} \leq \sum_{k=0}^{\infty}\left\|c_{k}\right\| \leq \sum_{\substack{k=0 \\ 0 \leq l \leq k}}^{\infty}\left\|a_{k-l}\right\|\left\|b_{l}\right\|=\sum_{k=0}^{\infty}\left\|a_{k}\right\| \sum_{l=0}^{\infty}\left\|b_{l}\right\| .
$$

Replacing the rightmost sums by the respective infima taken over all possible representations (11) of $x$ and $y$, we obtain $\left\|\left|x y\left\|_{\lambda} \leq\right\|\right||x|\right\|_{\lambda}\left|\|y \mid\|_{\lambda}\right.$. The subadditivity and homogeneity of $\||\cdot|\|_{\lambda}$ are established in a similar fashion.

We now prove that

$$
\left\||| x\left|\left\|_{\lambda} \leq\right\| x\|\leq M\|\right|\right\| x \|_{\lambda} \quad(x \in \boldsymbol{A}) .
$$

Since \|\|$\cdot\|\|_{\lambda}$ is a pseudonorm, this will in particular show that $\|\mid \cdot\| \|_{\lambda}$ is a norm. First, note that any $x \in \boldsymbol{A}$ can be written as $x=x+0 s_{\lambda}+0 s_{\lambda}^{2}+\ldots$. It follows from this that $\|x\|_{\lambda} \leq\|x\|$. Next, observe that $\left\|s_{\lambda}^{k}\right\| \leq M$ for each $k \in \mathbb{N}$. Therefore if $x$ is given by (11), then $\|x\| \leq M \sum_{k=0}^{\infty}\left\|a_{k}\right\|$, and so $\|x\| \leq M\|\| x\|\|_{\lambda}$.

We now show that

$$
\left\|\mid r_{\lambda}\right\|_{\mu} \leq \frac{1}{\lambda-\omega} \quad(\mu \in[\lambda,+\infty))
$$


Fix $\mu \in[\lambda,+\infty)$. Since $r_{\lambda}=r_{\mu}+(\mu-\lambda) r_{\mu} r_{\lambda}$ and because of (12), we have

$$
\begin{aligned}
\|\left|r_{\lambda}\right|||_{\mu} & \leq\left\|\left.|| r_{\mu}||\right|_{\mu}+(\mu-\lambda)||\left|r_{\mu}\right|||_{\mu}||\left|r_{\lambda}\right|\right\|_{\mu} \\
& \leq \frac{1}{\mu-\omega}+\frac{\mu-\lambda}{\mu-\omega}||\left|r_{\lambda} \|\right|_{\mu},
\end{aligned}
$$

whence

$$
\left(1-\frac{\mu-\lambda}{\mu-\omega}\right)\left\|\mid r_{\lambda}\right\| \|_{\mu} \leq \frac{1}{\mu-\omega},
$$

yielding (14).

Now set

$$
|||x|||=\limsup _{\lambda \rightarrow \infty}|||x| \|_{\lambda} \quad(x \in \boldsymbol{A}) .
$$

In view of (13), $|\| \cdot \cdot|||$ is a well-defined submultiplicative norm on $\boldsymbol{A}$ which satisfies (9). From (14) it follows that (10) is fulfilled.

\section{Proof of Theorem 1}

We are now ready to give a proof of Theorem 1.

Proof of Theorem 1. Suppose that $T: L_{\omega}^{1}\left(\mathbb{R}^{+}\right) \rightarrow \boldsymbol{A}$ is a continuous homomorphism such that $T\left(\epsilon_{-\lambda}\right)=r_{\lambda}$ for each $\lambda \in(\omega,+\infty)$. A standard argument using, say, the Stone-Weierstrass theorem shows that the set $\left\{\epsilon_{-\lambda}: \lambda \in(\omega,+\infty)\right\}$ is linearly dense in $L_{\omega}^{1}\left(\mathbb{R}^{+}\right)$. Therefore $T$ is uniquely determined by its values at the functions $\epsilon_{-\lambda}(\lambda \in(\omega,+\infty))$. Taking into account (2), we see that $M \leq\|T\|$, where $M$ is defined in (3). In particular, $M$ is finite. Now-as a moment's reflection reveals - to complete the proof, it suffices to show that if, conversely, $M$ is finite, then there exists a continuous homomorphism $T: L_{\omega}^{1}\left(\mathbb{R}^{+}\right) \rightarrow \boldsymbol{A}$ such that $T\left(\epsilon_{-\lambda}\right)=r_{\lambda}$ for each $\lambda \in(\omega,+\infty)$ and $\|T\| \leq M$.

Suppose then that $M$ is finite. Let $\boldsymbol{B}_{0}$ be the subalgebra of $\boldsymbol{A}$ generated by all the $r_{\lambda}(\lambda \in(\omega,+\infty))$. In view of $(1), \boldsymbol{B}_{0}$ is commutative. Let $\boldsymbol{B}$ be the closure of $\boldsymbol{B}_{0}$ in $\boldsymbol{A}$. Clearly, $\boldsymbol{B}$ is a commutative Banach algebra. By virtue of Theorem 2, there exists a submultiplicative norm $\||\cdot|\|$ on $\boldsymbol{B}$ satisfying (9) and (10). Let $\left\{\lambda_{i}\right\}_{i \in \mathbb{N}}$ be a sequence in $(\omega,+\infty)$ diverging to infinity. For each $i \in \mathbb{N}$, let

$$
e_{i}=\lambda_{i} r_{\lambda_{i}} .
$$

We claim that $\left\{e_{i}\right\}_{i \in \mathbb{N}}$ is a metric approximate identity for $\boldsymbol{B}$ provided $\boldsymbol{B}$ is taken with the norm $|\|\cdot|\||$. Indeed, in view of (10),

$$
||\left|e_{i}\right| \| \leq \frac{\lambda_{i}}{\lambda_{i}-\omega} \quad(i \in \mathbb{N}),
$$

so (5) is satisfied. Furthermore, for each $\mu \in(\omega,+\infty)$ and each $i \in \mathbb{N}$ with $\lambda_{i} \neq \mu$,

$$
e_{i} r_{\mu}=\frac{\lambda_{i}}{\lambda_{i}-\mu} r_{\mu}-\frac{1}{\lambda_{i}-\mu} e_{i} .
$$

Hence, for each $\mu \in(\omega,+\infty)$,

$$
\lim _{i \rightarrow \infty} e_{i} r_{\mu}=r_{\mu} .
$$

Since any $x \in \boldsymbol{B}_{0}$ is a linear combination of elements of the form $r_{\mu_{1}} \ldots r_{\mu_{k}}$ $\left(\mu_{1}, \ldots, \mu_{k} \in(\omega,+\infty)\right)$, it follows that

$$
\lim _{i \rightarrow \infty} e_{i} x=x
$$


for each $x \in \boldsymbol{B}_{0}$. Now, taking into account that $\left\{e_{i}\right\}_{i \in \mathbb{N}}$ is bounded, we see that the last equality holds for each $x \in \boldsymbol{B}$. The claim is established.

Let $\rho$ be the regular representation of $\boldsymbol{B}$ on $\boldsymbol{B}$. Clearly, $\left\{\rho_{r_{\lambda}}\right\}_{\lambda \in(\omega,+\infty)}$ is a pseudo-resolvent in $\mathcal{L}(\boldsymbol{B})$. Using (1), it is easy to verify that all the $\rho_{r_{\lambda}}(\lambda \in$ $(\omega,+\infty))$ have a common null space $\mathcal{N}$ and a common range $\mathcal{R}$. Restating the fact that $\left\{e_{i}\right\}_{i \in \mathbb{N}}$ is an approximate identity for $\boldsymbol{B}$ as

$$
\lim _{i \rightarrow \infty} \lambda_{i} \rho_{r_{\lambda_{i}}} x=x \quad(x \in \boldsymbol{B}),
$$

we immediately find that $\mathcal{N}$ is zero and $\mathcal{R}$ is dense in $\boldsymbol{B}$. Therefore $\left\{\rho_{r_{\lambda}}\right\}_{\lambda \in(\omega,+\infty)}$ is the resolvent of a densely defined linear operator on $\boldsymbol{B}$. More specifically, there exists a linear operator $A$ defined on $\mathcal{R}$, taking values in $\boldsymbol{B}$, such that, for each $\lambda \in$ $(\omega,+\infty)$, the inverse of $\rho_{r_{\lambda}}$ coincides with $\lambda \operatorname{id}_{\boldsymbol{B}}-A$, where $\mathrm{id}_{\boldsymbol{B}}$ denotes the identity operator on $\boldsymbol{B}$ (cf. [6, §8, Sec. 4, Thm. 1]). By (10) and the Hille-Yosida theorem (cf. $[6, \S 9$, Sec. 7$]$ ), $A$ is the generator of a one-parameter strongly continuous semigroup $\left\{S_{t}\right\}_{t \in[0,+\infty)}$ on $\boldsymbol{B}$ satisfying

$$
\left\|\left|S_{t} \|\right| \leq e^{\omega t} \quad(t \in[0,+\infty)),\right.
$$

where, of course, ||$|R|\left\|\left|=\sup _{\||| x|| \leq 1}\right|\right\| R x \mid \|$ for any $R \in \mathcal{L}(\boldsymbol{B})$. Moreover,

$$
\rho_{r_{\lambda}}=\int_{\mathbb{R}^{+}} \epsilon_{-\lambda}(t) S_{t} d t \quad(\lambda \in(\omega,+\infty)),
$$

where the integral is taken in the sense of the strong operator topology. For each $\varphi \in L_{\omega}^{1}\left(\mathbb{R}^{+}\right)$, define an element $S(\varphi)$ of $\mathcal{L}(\boldsymbol{B})$ by setting

$$
S(\varphi)=\int_{\mathbb{R}^{+}} \varphi(t) S_{t} d t
$$

It is easily verified that $S: \varphi \mapsto S(\varphi)$ is a homomorphism of $L_{\omega}^{1}\left(\mathbb{R}^{+}\right)$into $\mathcal{L}(\boldsymbol{B})$. By (15)

$$
||\left|S(\varphi)\|\mid \leq\| \varphi \|_{1, \omega} \quad\left(\varphi \in L_{\omega}^{1}\left(\mathbb{R}^{+}\right)\right)\right.
$$

and by $(16)$

$$
S\left(\epsilon_{-\lambda}\right)=\rho_{r_{\lambda}} \quad(\lambda \in(\omega,+\infty)) .
$$

Combining (17) and (18) with the fact that $\left\{\epsilon_{-\lambda}: \lambda \in(\omega,+\infty)\right\}$ is linearly dense in $L_{\omega}^{1}\left(\mathbb{R}^{+}\right)$, we see that, for each $\varphi \in L_{\omega}^{1}\left(\mathbb{R}^{+}\right), S(\varphi)$ belongs to the closed linear span of $\left\{\rho_{r_{\lambda}}: \lambda \in(\omega,+\infty)\right\}$. But, by Proposition 1, $\rho_{\boldsymbol{B}}$ is closed in $\mathcal{L}(\boldsymbol{B})$. Therefore $S(\varphi)$ is a member of $\rho_{\boldsymbol{B}}$. Since, again by Proposition 1, $\rho$ is isometry, it follows that, for each $\varphi \in L_{\omega}^{1}\left(\mathbb{R}^{+}\right)$, there exists a unique element $T(\varphi)$ of $\boldsymbol{B}$ such that

$$
\rho_{T(\varphi)}=S(\varphi) \quad \text { and } \quad\|T(\varphi)\|\|=\| S(\varphi)\|\| .
$$

Clearly, $T: \varphi \mapsto T(\varphi)$ is a homomorphism of $L_{\omega}^{1}\left(\mathbb{R}^{+}\right)$into $\boldsymbol{B}$. Moreover, by (18),

$$
T\left(\epsilon_{-\lambda}\right)=r_{\lambda} \quad(\lambda \in(\omega,+\infty)) .
$$

In view of (9) and (17), for each $x \in \boldsymbol{B}$ and each $\varphi \in L_{\omega}^{1}\left(\mathbb{R}^{+}\right)$,

$$
\begin{aligned}
\|T(\varphi) x\| & \leq M\||T(\varphi) x\||\leq M\||\|T(\varphi) \mid\|\|\| x\|\| \\
& =M\||| S(\varphi)\|\|\| x\|\leq \leq M\| \varphi\left\|_{1, \omega}\right\| x\|\| \leq M\|\varphi\|_{1, \omega}\|x\| .
\end{aligned}
$$

Hence $\|T\| \leq M$, which completes the proof. 


\section{REFERENCES}

1. W. Arendt, Vector-valued Laplace transforms and Cauchy problems, Israel J. Math. 59 (1987), 327-352.

2. H. F. Bohnenblust and S. Karlin, Geometrical properties of the unit sphere of Banach algebras, Ann. of Math. (2) 62 (1955), 217-229.

3. B. Hennig and F. Neubrander, On representations, inversions, and approximations of Laplace transforms in Banach spaces, Applicable Anal. 49 (1993), 151-170.

4. J. Kisyński, personal communication.

5. F. Neubrander, The Laplace-Stieltjes transform in Banach spaces and abstract Cauchy problems, Evolution equations, control theory, and biomathematics: proceedings of the Han-surLesse conference (Ph. Clément and G. Lumer, eds.), Lecture Notes in Pure and Applied Mathematics, vol. 155, Marcel Dekker, Basel, 1994, pp. 417-431.

6. K. Yosida, Functional Analysis, 5 ed., Springer-Verlag, Berlin, Heidelberg, New York, 1978.

Instytut Matematyki Stosowanej i Mechaniki, Uniwersytet Warszawski, Ul. Banacha 2, 02-097 Warszawa, Poland

E-mail address: wojtekch@appli.mimuw.edu.pl

Department of Computer Science, University of Adelaide, Adelaide, SA 5005, AusTRALIA

E-mail address: wojtek@cs.adelaide.edu.au 